\title{
Dictynna
}

Dictynna

Revue de poétique latine

$6 \mid 2009$

Varia

\section{Un frammento di una declamazione di Cicerone e due controversiae senecane}

\section{Emanuele Berti}

\section{Q OpenEdition}

\author{
Journals
}

Edizione digitale

URL: http://journals.openedition.org/dictynna/247

DOI: $10.4000 /$ dictynna.247

ISSN: 1765-3142

Notizia bibliografica digitale

Emanuele Berti, « Un frammento di una declamazione di Cicerone e due controversiae senecane », Dictynna [En ligne], 6| 2009, mis en ligne le 29 novembre 2010, consulté le 10 décembre 2020. URL http://journals.openedition.org/dictynna/247 ; DOI : https://doi.org/10.4000/dictynna.247

Questo documento è stato generato automaticamente il 10 décembre 2020.

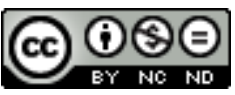

Les contenus des la revue Dictynna sont mis à disposition selon les termes de la Licence Creative Commons Attribution - Pas d'Utilisation Commerciale - Pas de Modification 4.0 International. 


\title{
Un frammento di una declamazione di Cicerone e due controversiae senecane
}

\author{
Emanuele Berti
}

1 La controversia 1, 4 della raccolta di Seneca il Vecchio Oratorum et rhetorum sententiae divisiones colores propone il caso dell'abdicatio di un figlio, ripudiato dal padre vir fortis ma mutilo delle mani perdute in battaglia, per non aver voluto sostituirsi a lui nell'uccidere la madre sorpresa in adulterio insieme all'amante, secondo quanto la legge permetteva ${ }^{1}$. Giunto a trattare dei colores ${ }^{2}$ in difesa del giovane, Seneca introduce la seguente annotazione : Sen. contr. 1, 4, 7 :

Color pro adulescente unus ab omnibus qui declamaverunt introductus est: non potui occidere, ex illa Ciceronis sententia tractus quam in simili controversia dixit, cum abdicaretur is qui adulteram matrem occidendam acceperat et dimiserat: ter non ***? 3

2 Tale testimonianza, che preserva l'unico frammento a noi noto di una declamazione ciceroniana, presenta molteplici motivi di interesse, ma suscita anche diversi interrogativi. La prima questione che si pone è naturalmente quella dell'attendibilità della notizia, se siamo cioè davvero in presenza di un'autentica sententia tratta da una controversia di Cicerone ; seri dubbi in proposito erano avanzati da Janet Fairweather, che così si esprimeva : "perhaps we should not lay too much weight on this piece of school tradition, originating as it may in the realms of Ciceronian pseudepigrapha, or simply in some rethorician's fanciful wish to ascribe a time-honoured color to the fount of eloquence himself. But it is not totally implausible that some memory of the nature of Cicero's declamations, at least his late ones, should have been preserved" 4 . Prima di tutto dobbiamo dunque chiederci se effettivamente Cicerone poteva aver composto delle controversiae come quella menzionata da Seneca, e in caso affermativo, se è possibile che queste (o comunque parti di esse) avessero avuto qualche forma di circolazione, fino a essere conosciute e diventare oggetto di imitazione da parte dei retori della prima età imperiale. 
3 Sulle declamazioni di Cicerone possediamo un buon numero di informazioni, che consentono di farci un quadro assai preciso ${ }^{5}$. È innanzitutto lo stesso Cicerone che rievocando nel Brutus gli inizi della sua carriera oratoria, ricorda di aver praticato assiduamente questo genere di esercizio, anche insieme ad altri oratori suoi coetanei come Marco Pupio Pisone Frugi e Quinto Pompeo Bitinico, declamando in latino, ma prevalentemente in greco, dato che dalla Grecia provenivano i migliori maestri di retorica, e questa era la lingua tradizionalmente adottata nell'insegnamento (cfr. Cic. Brut. 310-11 huic ego doctori [cioè al filosofo stoico Diodoto, uno dei maestri di Cicerone] et eius artibus variis atque multis ita eram tamen deditus ut ab exercitationibus oratoriis nullus dies vacuus esset. Commentabar declamitans - sic enim nunc loquuntur ${ }^{6}$ - saepe cum M. Pisone et cum Q. Pompeio aut cum aliquo cottidie, idque faciebam multum etiam Latine sed Graece saepius, vel quod Graeca oratio plura ornamenta suppeditans consuetudinem similiter Latine dicendi adferebat, vel quod a Graecis summis doctoribus, nisi Graece dicerem, neque corrigi possem neque doceri) $)^{7}$. Ulteriori notizie sono riferite nel capitolo iniziale del De rhetoribus di Svetonio, dove l'autore, nel tracciare un breve profilo storico dell'introduzione dell'arte retorica a Roma, ricorda non solo le declamazioni giovanili di Cicerone, fino all'epoca della sua pretura (nel 66 a.C.) ${ }^{8}$, ma anche quelle svolte in età avanzata, quando egli ebbe come allievi i consoli del 43 a.C., Aulo Irzio e Vibio Pansa (cfr. Suet. rhet. 25, 3 Cicero ad praeturam usque etiam Graece declamitavit, Latine vero senior quoque et quidem cum consulibus Hirtio et Pansa, quos discipulos et grandis praetextatos vocabat $)^{9}$; e a quest'ultima circostanza fa riferimento anche Seneca il Vecchio, che nella praefatio al l. I delle Controversiae vi allude mediante la stessa espressione grandes praetextati, lamentando di non avere potuto assistere a tali sessioni declamatorie, poiché tenuto lontano da Roma dal furor delle guerre civili (cfr. Sen. contr. 1 praef. 11 ne Ciceronem quidem aetas mihi eripuerat, sed bellorum civilium furor, qui tunc orbem totum pervagabatur, intra coloniam meam me continuit; alioqui in illo atriolo, in quo duos grandes praetextatos ait secum declamare, potui adesse).

4 Dobbiamo dunque distinguere due fasi nell'attività declamatoria di Cicerone, distanti non solo cronologicamente, ma anche, per così dire, concettualmente. Da un lato vi sono le declamazioni che risalgono al periodo dei suoi studi retorici, sotto la guida di maestri soprattutto greci, praticate a scopo di esercizio e in preparazione all'impegno forense (anche se è possibile che Cicerone abbia continuato a seguire questa routine anche in età più matura, quando era un oratore già affermato, come si deduce sia dalla testimonianza di Svetonio, sia da un accenno nel proemio delle Tusculanae disputationes, su cui torneremo più avanti, in cui egli afferma di aver coltivato la declamazione più a lungo di chiunque altro $^{10}$ ). In questo Cicerone appare essersi attenuto a quella che già nei primi decenni del I sec. a.C. era una prassi abituale per i giovani studenti di retorica : lo attesta ad esempio un passo del dialogo De oratore (la cui data drammatica si colloca nel 91 a.C.), in cui Crasso, trattando dell'exercitatio, menziona tra gli altri un tipo di esercizio consistente nel dibattere casi fittizi simili a quelli del foro, che corrisponde in tutto e per tutto alla controversia (anche se nel contesto non è usato questo termine, né l'altro di declamatio) ${ }^{11}$. Si tratta dunque di declamazioni che avevano una finalità esclusivamente didattica e propedeutica, ed è per questo assai improbabile che esse siano fuoriuscite da una dimensione strettamente privata.

5 Dall'altro lato, vi sono le declamazioni degli ultimi anni della sua vita, quando lo stesso Cicerone si adattò a fare da 'maestro' ad alcuni importanti personaggi di rango consolare, come Irzio, Pansa e anche altri, declamando insieme a loro e trovando in 
questo, oltre che un modo di impiegare utilmente il proprio otium e di tenere in esercizio la sua facoltà oratoria in un periodo in cui le vicende politiche l'avevano allontanato dalla pratica diretta del foro, anche un momento di svago in mezzo alle tempeste e alle amarezze della vita politica. È quanto emerge da diversi passi dell'epistolario ciceroniano, che integrano le notizie offerte da Svetonio e Seneca il Vecchio e consentono di precisarne le circostanze; da una serie di lettere ad Attico apprendiamo così che quanto narrato dai due succitati autori deve riferirsi a un soggiorno nella villa di Puteoli (Pozzuoli) nell'aprile-maggio del 44 a.C., poco dopo le Idi di marzo, quando Cicerone ebbe suoi ospiti fra gli altri i consoli designati Irzio e Pansa (cfr. Cic. Att. 14, 11, 2 [lettera del 21 aprile] hic mecum Balbus, Hirtius, Pansa). Alle declamazioni tenute insieme a questi ultimi Cicerone accenna in realtà soltanto fugacemente, senza scendere nei dettagli, in una lettera del 22 aprile (cfr. Cic. Att. 14, 12, 2 haud amo vel hos designatos, qui etiam declamare me coegerunt, ut ne apud aquas quidem acquiescere liceret $)^{12}$; mentre in nessun punto della sua corrispondenza ricorre l'espressione, comune sia a Svetonio che a Seneca, grandes praetextati, che dunque sarà stata tratta da una lettera o da un'altra opera perduta, dove Cicerone forniva forse particolari più precisi sui caratteri e le modalità di questi esercizi declamatòri.

Più interessante per noi è un gruppo di tre epistole risalenti all'estate del 46 a.C., nelle settimane successive alla battaglia di Tapso, quando, in seguito al trionfo di Cesare nella guerra civile, Cicerone si trovò praticamente estromesso dalla vita politica, e si ritirò per un periodo nella sua tenuta di Tuscolo, accompagnato dallo stesso Irzio e dal genero Publio Cornelio Dolabella (entrambi fra i più importanti partigiani di Cesare). In due lettere all'amico Lucio Papirio Peto, scritte a pochi giorni di distanza l'una dall'altra, Cicerone racconta dunque di avere preso Irzio e Dolabella come suoi discipulos dicendi, per istruirli nell'arte della declamazione (cfr. Cic. fam. 9, 16, 7 Hirtium ego et Dolabellam dicendi discipulos habeo, cenandi magistros; puto enim te audisse, si forte ad vos omnia perferuntur, illos apud me declamitare, me apud illos cenitare), e di avere aperto a loro beneficio una specie di scuola di retorica (ludus), che andava in qualche modo a compensare il forzato abbandono delle cause giudiziarie e la perdita del regnum forense (cfr. Cic. fam. 9, 18, 1 cum essem otiosus in Tusculano, propterea quod discipulos obviam miseram ut eadem me quam maxime conciliarent familiari suo, accepi tuas litteras plenissimas suavitatis; ex quibus intellexi probari tibi meum consilium, quod, ut Dyonisius tyrannus, cum Syracusis pulsus esset Corinthi dicitur ludum aperuisse ${ }^{13}$, sic ego sublatis iudiciis, amisso regno forensi ludum quasi habere coeperim). Nel seguito di questa seconda lettera Cicerone si dilunga a enumerare $\mathrm{i}$ vantaggi di tale risoluzione, consistenti in primo luogo in ragioni di opportunità politica (rendendosi disponibile a fare da maestro a Irzio e Dolabella, Cicerone sperava di ottenere il loro aiuto per conciliarsi la benevolenza di Cesare), e poi nella possibilità di riprendere le esercitazioni retoriche, da lungo tempo abbandonate, traendone giovamento non solo per la sua facultas orationis, ma anche per la sua salute ${ }^{14}$ ; la lettera si conclude infine con una nota scherzosa, quando Cicerone indica come terzo motivo che lo ha indotto ad aprire la scuola le prelibatezze che egli ha modo di gustare frequentando Irzio, noto buongustaio ${ }^{15}$. Forse ancora più significativa è una terza lettera, scritta questa volta da Roma a Publio Volumnio Eutrapelo, non datata ma risalente senza dubbio a quegli stessi giorni, dopo il rientro di Cicerone da Tuscolo nella capitale $^{16}$; in risposta al rammarico evidentemente manifestato dall'amico per non aver potuto assistere alle sue declamazioni e ricevere i suoi insegnamenti al pari di Irzio, Cicerone lo rassicura affermando che non si tratta di una perdita grave, dato che è lui per primo a provare disgusto per se stesso, e si serve della citazione di alcuni versi del 
Filottete di Accio per esprimere l'amara consapevolezza che le declamazioni, in quanto indirizzate alla trattazione di casi fittizi e immaginari, sono come armi spuntate, che non producono nessun effetto concreto, né tanto meno possono procurare alcuna gloria : cfr. Cic. fam. 7, 33, 1 quod declamationibus nostris cares, damni nihil facis. Quod Hirtio invideres nisi eum amares, non erat causa invidendi, nisi forte ipsius eloquentiae magis quam quod me audiret invideres. Nos enim plane, mi suavissime Volumni, aut nihil sumus, aut nobis quidem ipsis displicemus gregalibus illis quibus te plaudente vigebamus amissis, ut etiam, si quando aliquid dignum nostro nomine emisimus, ingemescamus quod haec 'pinnigero, non armigero in corpore tela exerceantur', ut ait Philoctetes apud Accium, 'abiecta gloria ${ }^{17}$.

7 Al di là del significativo riconoscimento della futilità dell'esercizio declamatorio, che in nessun caso può valere come surrogato della reale pratica oratoria, il passo ci interessa per la frase si quando aliquid dignum nostro nomine emisimus (se con essa Cicerone vuole alludere, come pare probabile, alle declamazioni menzionate all'inizio della lettera, e non in generale all'attività letteraria svolta in questo periodo). Il verbo emitto, quando usato in relazione a scritti o opere letterarie, contiene infatti sempre l'idea della pubblicazione e divulgazione dell'opera stessa ${ }^{18}$; le parole di Cicerone sembrano dunque implicare che, nonostante la natura certamente privata di queste sue declamazioni, esse fossero destinate anche a una circolazione presso un pubblico più ampio. La conferma potrebbe venire da un'interessante testimonianza di Quintiliano, che nel l. VIII dell'Institutio oratoria, trattando del vitium del pleonasmo, cita un'espressione di una declamazione di Irzio, con la relativa critica ad essa mossa da Cicerone : cfr. Quint. inst. 8, 3, 54 emendavit hoc etiam urbane in Hirtio Cicero: cum is apud ipsum declamans filium a matre decem mensibus in utero latum esse dixisset, 'quid? aliae' inquit 'in perula solent ferre ?'19. L'occasione a cui si fa qui riferimento è quasi certamente la stessa di cui Cicerone parla nelle lettere sopra ricordate ${ }^{20}$; e anche se la provenienza della battuta citata dal retore spagnolo non è determinabile con sicurezza ${ }^{21}$, questo sembra avvalorare l'ipotesi che le declamazioni composte non solo da Cicerone, ma anche dai suoi allievi in quella specie di scuola aperta a Tuscolo (oppure due anni dopo a Pozzuoli), avessero avuto una almeno parziale registrazione scritta e una diffusione pubblica (non sappiamo in quale forma, forse come raccolta di estratti o magari di 'appunti' ricavati dall'insegnamento di Cicerone), tanto che la loro memoria poteva essersi conservata nelle generazioni successive, almeno fino all'epoca di Quintiliano. Se è così, aumentano le probabilità, pur senza poterne avere la certezza assoluta, che anche nel caso della sententia trasmessa da Seneca il Vecchio ci troviamo davanti a un frammento autentico di una declamazione ciceroniana ${ }^{22}$.

8 Alla questione dell'autenticità del frammento si lega anche l'altra della natura delle declamazioni svolte da Cicerone. In contr. 1, 4, 7 Seneca parla di una controversia, e dà anche un sommario resoconto del thema (...cum abdicaretur is qui adulteram matrem occidendam acceperat et dimiserat) ; questo appare però in contrasto con le notizie offerte dallo stesso Seneca nell'excursus sulla storia della declamazione romana nella praefatio al 1. I delle Controversiae, dove si afferma esplicitamente che Cicerone non declamava controversiae, dato che questo tipo di esercizio sarebbe stato introdotto in un'epoca successiva (cfr. Sen. contr. 1 praef. 12 declamabat autem Cicero non quales nunc controversias dicimus, ne tales quidem quales ante Ciceronem dicebantur, quas thesis vocabant. Hoc enim genus materiae quo nos exercemur adeo novum est, ut nomen quoque eius novum sit. Controversias nos dicimus; Cicero causas vocabat). Come tuttavia hanno riconosciuto gli studiosi che si sono occupati di questo importante brano, qui Seneca ha fatto parecchia confusione, mescolando informazioni terminologiche, che si possono ritenere corrette, 
con notizie di carattere storico, che contengono invece diverse inesattezze ${ }^{23}$. Così egli ha ragione nel dire che il termine controversia, come designazione tecnica degli esercizi declamatòri del genere giudiziale, entra in uso soltanto in età post-ciceroniana, mentre in precedenza essi erano definiti causa ${ }^{24}$; e che d'altra parte le declamazioni praticate da Cicerone avessero il nome di causae è da lui stesso affermato in un passo del proemio delle Tusculanae, a cui si è già fatto riferimento (cfr. Cic. Tusc. 1, 7 ut enim antea declamitabam causas, quod nemo me diutius fecit, sic haec mihi nunc senilis est declamatio). Ciò in cui Seneca sbaglia è nel ritenere che al mutamento di nome corrispondesse anche l'adozione di un novum genus materiae, che cioè causae e controversiae fossero qualcosa di sostanzialmente diverso tra di loro; in realtà, tutto ciò che sappiamo delle declamazioni del periodo repubblicano mostra che esiste una notevole continuità, almeno nei soggetti trattati, fra queste e quelle di epoca più tarda ${ }^{25}$. Nella sua corrispondenza Cicerone non specifica quali fossero la natura e i temi dei suoi esercizi retorici (con l'eccezione di una lettera ad Attico del marzo del 49 a.C., in cui egli elenca i titoli di alcune theseis di argomento politico che si esercitava a dibattere ; ma si tratta di un caso particolare, una scelta determinata dalle circostanze storiche del momento) ${ }^{26}$; in questo ci viene allora in aiuto la testimonianza senecana, che preserva un dettaglio importante dal punto di vista storico-letterario, documentando che, in qualunque modo l'autore le chiamasse, le declamazioni ciceroniane erano senz'altro definibili come controversiae, in tutto e per tutto analoghe a quelle svolte dai retori dell'età imperiale (il thema cui Seneca fa cenno - il figlio che riceve in consegna dal padre la madre adultera con l'ordine di ucciderla, ma la lascia andare - è praticamente lo stesso della contr. 1, 4, ma è anche simile alla contr. 7, 1, come vedremo fra poco). A sostegno di questa conclusione possiamo ancora addurre il già citato passo di Quint. inst. 8, 3, 54, dove si parla della declamazione di Irzio ; anche se Quintiliano non dà indicazioni sul soggetto di quest'ultima, il contenuto della frase di Irzio criticata da Cicerone (filium a matre decem mensibus in utero latum esse) risulta affine a una sententia del retore Albucio Silo che si legge in Sen. contr. 7, 4, 1 (ergo tu, adulescens, matri tuae ne decem mensum quidem alumenta reddes?), tratta da una controversia che propone il caso di un figlio diviso tra l'obbligo di andare a riscattare il padre rapito dai pirati, e quello di rimanere a casa ad assistere la madre cieca ${ }^{27}$. Appare pertanto plausibile che la frase di Irzio derivasse da una controversia simile a questa, in cui una madre rivendicava i propri diritti di fronte a un atto di ingratitudine del figlio ; e addirittura si potrebbe arrivare a supporre che si tratti della stessa controversia menzionata da Seneca in contr. 1, 4, 7, dove si ha una non dissimile situazione di contrasto fra il dovere del figlio di obbedire all'ordine del padre, e il suo affetto nei confronti della madre. Si conferma così la genuinità della notizia senecana, che anche sotto questo aspetto si rivela fonte di informazioni preziose, che hanno una rilevanza più generale per la storia dello sviluppo del genere declamatorio a Roma.

9 L'ultimo problema concerne il testo della sententia ciceroniana, che come si può vedere è andata purtroppo perduta, tranne le due prime parole (ter non...), nella tradizione manoscritta dell'opera di Seneca. In una breve nota esplicativa al passo senecano, Michael Winterbottom suggeriva la possibilità che l'avverbio ter introducesse il modulo di ascendenza epica della triplicazione dei tentativi : tre volte il figlio tenta di uccidere la madre, tre volte è costretto a desistere ${ }^{28}$. Questo è senz'altro possibile (anche se in nessuna delle sententiae della nostra controversia, che a detta di Seneca sarebbero ispirate a quella di Cicerone, ricorre qualche cosa di analogo ${ }^{29}$; ma quanto resta del frammento è troppo poco per poter formulare qualunque fondata supposizione ${ }^{30}$. 
Tuttavia le parole con cui Seneca introduce la citazione consentono almeno di ricostruire il senso generale della sententia: appare così che essa dovesse essere incentrata intorno al motivo del 'non potui occidere', svolgesse cioè l'idea dell'incapacità del figlio di compiere quell'uccisione che gli veniva richiesta dal padre.

Ora, proprio questo motivo, declinato in maniera diversa dai vari declamatori, costituisce, a quel che possiamo vedere, il cardine della difesa del figlio non solo nella contr. 1, 4, ma anche in un'altra controversia della raccolta senecana, la 7, 1, che presenta un caso sostanzialmente analogo, nella sua questione di fondo, sia alla 1, 4, sia alla declamazione svolta da Cicerone : anche in questa seconda controversia abbiamo infatti un figlio che viene incaricato dal padre di eseguire la condanna a morte da lui decretata nei confronti di un congiunto (qui il fratello anziché la madre), ma che rinuncia a farlo e subisce per questo l'abdicatio ; anche se in questo caso il thema propone un'evidente accentuazione degli elementi romanzeschi, con la consegna del fratello a un navigium exarmatum e il suo insperato salvataggio, la sua successiva trasformazione in pirata e la cattura e liberazione del padre (che scoprendo in questo modo la disobbedienza dell'altro figlio, gli infligge l'abdicatio) ${ }^{31}$. Le due controversiae presentano dunque nella parte difensiva (alla quale, al contrario di quanto accade nella contr. 1, 4, dove essa è confinata quasi esclusivamente nei colores, viene dato nella contr. 7, 1 lo spazio più ampio) evidenti punti di contatto, che in ultima analisi si possono ricondurre a quel color - 'non potui occidere' - ispirato dalla sententia di Cicerone, e che vale la pena osservare più nel dettaglio.

11 Nella contr. 1, 4, alla citazione della sententia ciceroniana Seneca associa immediatamente lo sviluppo del declamatore Porcio Latrone ${ }^{32}$, in cui il motivo del 'non potui occidere' si lega alla descrizione dello stupor che coglie il figlio alla vista dell'inopinato spectaculum della madre adultera: cfr. contr. 1, 4, 7 Latro descripsit stuporem totius corporis in tam inopinati flagitii spectaculo et dixit: pater, tibi manus defuerunt, mihi omnia. Et cum oculorum caliginem, animi defectionem, membrorum omnium torporem descripsisset, adiecit: antequam ad me redeo, exierunt. Questo genere di descrizione, con gli elementi topici dell'ottenebramento della vista, il torpore di tutte le membra, il mancamento dei sensi, sembra essere una specialità di Latrone, che la riusa come pezzo forte ed efficace color difensivo in varie controversiae $e^{33}$; è pertanto abbastanza ovvio ritrovare una simile descriptio, ancora legata al color del 'non potui occidere', nella contr. 7, 1, come Seneca rileva nell'esposizione della divisio di Latrone (cfr. contr. 7, 1, 17 fatebor, inquit, quod fortasse offensurum est aures <vestras $>$ : patri parere volui ; fratrem occidere non potui. Obortae sunt subito tenebrae, deriguit animus, sublapsum est intercepto spiritu corpus), poi di nuovo, più esplicitamente, all'inizio della sezione dei colores: cfr. contr. 7, 1, 20 Latro illum introduxit colorem rectum in narratione, quo per totam actionem usus est : non potui occidere. Et cum descripsisset tingenti ${ }^{34}$ spiritu titubantem et inter cogitationem fratris occidendi concidentem, dixit: noverca, aliud quaere in privignum tuum crimen : hic parricidium non potest facere (degna di nota è anche la definizione di tale color come rectus, un epiteto che, insieme a simplex, Seneca applica a quei colores che si fanno apprezzare per la loro semplicità e immediatezza) ${ }^{35}$.

Un diverso sviluppo del color è dato nella contr. 1, 4 dall'altro retore Albucio Silo ${ }^{36}$, che spiega l'incapacità di uccidere del figlio con la mitezza della sua indole, facendo riferimento in generale al motivo della diversità degli ingenia, che rende alcune persone inette a determinate attività o imprese : cfr. contr. 1, 4, 8 Albucius non narravit, sed hoc colore egit ab initio usque ad finem : ego me defendere debeo? Si quid mihi obiectum erit, aut 
negabo aut excusabo. Si quid exegeris maius viribus meis, dicam : ignosce, non possum. Ignoscit filio pater navigationem recusanti, si non fert mare; ignoscit non sequenti castra, si non potest, quamvis pater ipse militaris sit: non possum occidere. Lo stesso tipo di approccio si ritrova anche nella contr. 7, 1, negli interventi di svariati declamatori ; accennato di nuovo nella divisio di Latrone ${ }^{37}$, esso è svolto più compiutamente, in connessione con l'altro motivo della pietas fraterna, da Giunio Gallione ${ }^{38}$, in un pezzo dal tono retoricamente assai sostenuto, ricco di figure di parola (tricolon e parallelismo, anafora, asindeto, omoteleuto, chiasmo), e chiuso da un'efficace sententia, che propone l'immagine paradossale del 'buon pirata', incapace di uccidere ${ }^{39}$ : cfr. contr. 7, 1, 12-13 non potui fratrem occidere: idem timuimus, idem doluimus, idem flevimus, eundem patrem habuimus, eandem matrem, eandem novercam. Mitioris natura pectoris sum, mollioris animi. Non idem omnibus mortalibus natura tribuit ingenium : animus durior est illius, illius clementior. Apud piratas quoque invenitur qui non possit occidere. Al brano di Gallione è probabilmente ispirato il successivo estratto del retore Musa $^{40}$, che ne riprende non solo il senso generale, ma anche alcune espressioni particolari (cfr. contr. 7, 1, 14 obicis mihi molliorem animum: alius mitior est, plus quam debet, alius saevior quam necesse est, mediis alius adfectibus inter utrumque positus totus in sua potestate est. Quidam et accusare et damnare possunt et occidere; quidam tam mites sunt, ut non possint in caput ne testimonium quidem dicere. Non possum hominem occidere: hoc vitium et apud piratas invenitur); ma Musa sviluppa ulteriormente la sua trattazione in direzione del topos filosofico della diversità delle scelte di vita, un ben noto luogo comune di matrice diatribica (per il quale basti pensare all'ode 1, 1 di Orazio), che però qui si inserisce in maniera alquanto forzata, svolto come un pezzo retorico indipendente ${ }^{41}$; e la conclusione della sequenza è affidata a un exemplum storico, che si distingue per la sua rarità e ricercatezza, ma che appare anch'esso assai poco pertinente al contesto ${ }^{42}$. Nelle mani di un declamatore come Musa, che spesso Seneca prende di mira per il suo dubbio gusto e la sua incontinenza espressiva ${ }^{43}$, il semplice color 'non potui occidere' si dilata in una lunga tirata retorica, in buona parte avulsa dal tema della controversia, contrassegnata dalla trattazione del locus communis e coronata dall'immancabile exemplum.

Una linea ancora diversa è scelta infine da Cestio Pio ${ }^{44}$, che nella contr. 1, 4 imposta il suo color sul contrasto fra pater e mater, ovvero sullo scontro (reso retoricamente mediante una triplice antitesi) fra la richiesta del padre di uccidere e quella della madre, ugualmente urgente, di essere risparmiata, con il prevalere infine nel figlio delle istanze dell'affetto materno : cfr. contr. 1, 4, 9 Cestius hoc colore egit : prosiluit, inquit, protinus mater et amplexu suo manus meas alligavit. Ago confusioni meae gratias, quod nihil in illo cubiculo vidi praeter matrem et patrem. Pater rogabat ut occiderem, mater ut viveret; pater ne nocens impunita esset, mater ut ego innocens essem; pater recitabat legem de adulteris, mater de parricidis. Et ultimam sententiam dixit: occidere matrem si turpe est noluisse, non potui ${ }^{45}$. Nella contr. 7, 1 la madre ovviamente non c'è, visto che, secondo il thema, essa è morta e il suo posto è stato preso dalla noverca (indicata da molti declamatori come la vera artefice della condanna del figlio ${ }^{46}$; ma Cestio trova il modo di recuperare ugualmente il motivo del contrasto pater / mater, immaginando che il figlio, mentre si reca a dare esecuzione alla condanna del fratello, si trovi a passare davanti al sepolcro della madre : cfr. contr. 7, 1, 21 Cestius colore alio usus est : transiebamus, inquit, secundum matris sepulchrum: invocare coepit manes eius; motus sum. Et puerili sensu colorem transcucurrit: quid facerem?, inquit; occidere pater iubebat, mater vetabat. Come si vede, Seneca è tutt'altro che tenero nei confronti di questo color, connotandolo come puerilis : ciò che l'autore rimprovera a Cestio è probabilmente proprio l'introduzione artificiosa 
di un'antitesi che, del tutto pertinente nella contr. 1, 4, non ha invece qui ragion d'essere; ma è come se il retore avesse voluto trasferire di proposito in quest'altra controversia un tratto già svolto brillantemente nella prima declamazione.

Non sappiamo come Cicerone sviluppasse il motivo del 'non potui occidere' nella sententia che Seneca il Vecchio riportava in contr. 1, 4, 7; ma sembra che questa abbia fornito ai nostri declamatori un'importante traccia, seguendo la quale essi hanno provveduto ad adattare lo stesso color, in tutte le varianti che abbiamo osservato, a due controversiae distinte, e diverse anche da quella in cui esso originariamente si trovava. L'influenza dell'illustre modello ciceroniano, risalente cioè a colui che era considerato l'auctor per eccellenza nel campo dell'eloquenza, agisce in maniera così pressante, che una sola sententia diviene il nucleo generativo su cui si fondano le due controversiae, determinando in esse la scelta del color principale, adottato dalla maggior parte dei retori, fino a improntare di fatto in entrambi i casi lo svolgimento dell'intera declamazione (da questo punto di vista non ha nemmeno molta importanza se il frammento citato da Seneca fosse autentico : ciò che conta è che i declamatori fossero convinti di avere a che fare con un'originale sententia tratta da una controversia di Cicerone).

Quello testimoniato da Seneca costituisce dunque un piccolo ma significativo esempio dell'aemulatio Ciceronis e del 'culto' riservato all'Arpinate all'interno delle scuole di retorica, da parte di quei declamatori che, pur dediti a un genere che era poco più di una parodia della grande oratoria politica e giudiziaria praticata da Cicerone, pur prediligendo una maniera stilistica lontana anni luce dall'equilibrato classicismo ciceroniano, continuavano a vedere in lui un insuperabile maestro e un modello da imitare e a cui ispirarsi ${ }^{47}$.

\section{NOTE}

1. Il thema della controversia (preceduto come di consueto dalla citazione delle leggi su cui il caso è basato) è il seguente : Adulterum cum adultera qui deprenderit, dum utrumque corpus interficiat, sine fraude sit. Liceat adulterium in matre et filio vindicare. Vir fortis in bello manus perdidit. Deprendit adulterum cum uxore ex qua filium adulescentem habebat, imperavit filio ut occideret : non occidit, adulter effugit. Abdicat filium. Sulle due leggi poste in capo alla controversia (nella seconda notiamo che filio è da intendere come dativo) cfr. S. F. Bonner, Roman Declamation in the Late Republic and Early Empire, Liverpool 1949, pp. 119 ss.; sul procedimento giuridico dell'abdicatio, uno dei più ricorrenti nella declamazione latina, cfr. da ultimo M. Lentano, 'Un nome più grande di qualsiasi legge’. Declamazione latina e patria potestas, « Boll. Stud. Lat. » 35, 2005, pp. 558-89, pp. 572 ss. (con bibliografia).

2. I colores, che sono una delle tre sezioni in cui Seneca il Vecchio ripartisce il materiale all'interno della sua raccolta antologica, sono le 'coloriture' e interpretazioni date dal retore ai fatti della controversia, a sostegno della propria strategia accusatoria o difensiva. Sul significato del termine e sulla funzione dei colores nella declamazione cfr. il recente contributo di L. Calboli Montefusco, La funzione strategica dei colores nella pratica declamatoria, in Ead. (ed.), Papers on Rhetoric VIII. Declamation, Roma 2007, pp. 157-77. 
3. Il testo di Seneca il Vecchio è citato secondo l'edizione di L. Håkanson (L. Annaeus Seneca maior, Oratorum et rhetorum sententiae divisiones colores, recensuit L.H., Leipzig 1989).

4. Cfr. J. Fairweather, Seneca the Elder, Cambridge 1981, p. 122.

5. Le testimonianze in tal senso sono raccolte e vagliate da S. F. Bonner, op. cit., pp. 27 ss.; J. Fairweather, Seneca the Elder cit., pp. 118 ss. ; Ead., The Elder Seneca and Declamation, in Aufstieg und Niedergang der römischen Welt, II 32, 1, Berlin-New York 1984, pp. 514-56, p. 548 e nn. 146-151.

6. L'inciso allude al fatto che all'epoca della giovinezza di Cicerone il verbo declamo (o declamito) e il corrispondente sostantivo declamatio non erano ancora entrati in uso come designazione tecnica dell'esercizio retorico consistente nell'esecuzione di discorsi fittizi specialmente del genere deliberativo (le suasoriae) o giudiziale (le controversiae), un significato che essi assumeranno solo a partire dalle ultime opere di Cicerone, poi più stabilmente nell'età imperiale. Per la storia del termine declamatio cfr. adesso W. Stroh, Declamatio, in B.-J. und J.-P. Schröder (hrsg.), Studium declamatorium. Untersuchungen zu Schulübungen und Prunkreden von der Antike bis zur Neuzeit, München-Leipzig 2003, pp. 5-34.

7. All'addestramento bilingue da lui seguito negli anni della sua formazione Cicerone allude ancora in off. 1, 1 ; si veda inoltre il frammento di una lettera a M. Titinio citato da Suet. rhet. 26, 1. Alla stessa pratica si atterrà anni dopo il figlio di Cicerone, come si ricava da una sua lettera dell'estate del 44 a.C. conservata nella raccolta delle familiares, in cui da Atene, dove si trovava per un viaggio di istruzione, egli informa il liberto Tirone sui suoi studi (cfr. Cic. fam. 16, 21, 5 praeterea declamitare Graece apud Cassium institui, Latine autem apud Bruttium exerceri volo). Cfr. F. Lechi, Latino e greco nelle scuole di retorica, in F. Bellandi-R. Ferri (a cura di), Aspetti della scuola nel mondo romano. Atti del convegno (Pisa, 5-6 dicembre 2006), Amsterdam 2008, pp. 9-27, pp. 10 ss. ; in generale sull'educazione retorica di Cicerone cfr. A. Corbeill, Rhetorical Education in Cicero's Youth, in J. M. May (ed.), Brill's Companion to Cicero. Oratory and Rhetoric, Leiden-Boston-Köln 2002, pp. 23-48, pp. 25 ss.

8. Questo dettaglio è probabilmente da porre in relazione con l'altra notizia riportata da Svetonio nel cap. 7 del De grammaticis, secondo cui nell'anno della sua pretura Cicerone frequentava la scuola del grammatico e retore M. Antonio Gnifone (cfr. Suet. gramm. 7, 3-4 [M. Antonius Gnipho] docuit autem et rhetoricam, ita ut cotidie praecepta eloquentiae traderet, declamaret vero nonnisi nundinis. Scholam eius claros quoque viros frequentasse aiunt, in his M. Ciceronem, etiam cum praetura fungeretur; cfr. anche Macrob. Sat. 3, 12, 8).

9. Si vedano le note di commento a tutto il passo in R. A. Kaster, Suetonius, De grammaticis et rhetoribus, ed. with a Translation, Introduction and Commentary by R.A.K., Oxford 1995, pp. 275 s. 10. Cfr. Cic. Tusc. 1, 7 (citato infra, p. 9).

11. Cfr. Cic. de orat. 1, 149 equidem probo ista - Crassus inquit - quae vos facere soletis, ut causa aliqua posita consimili causarum earum quae in forum deferuntur, dicatis quam maxime ad veritatem accommodate, con il commento ad l. di A. D. Leeman-H. Pinkster, M. Tullius Cicero, De oratore libri III, I Band : Buch I, 1-165, Kommentar von A.D.L. und H.P., Heidelberg 1981, pp. 245 ss.

12. Alla pratica di declamazioni non sembrano riferirsi due ulteriori accenni in due lettere scritte sempre da Puteoli nel maggio successivo (cfr. Cic. Att. 14, 20, 4 e 22, 1, dove Irzio è definito meus discipulus, ma da intendere probabilmente in senso politico : cfr. R. A. Kaster, op. cit., p. 276).

13. Cicerone allude alla vicenda del tiranno di Siracusa Dionisio il Giovane, il quale, cacciato dalla sua patria ad opera di Timoleone, si rifugiò a Corinto, dove per vivere aprì una scuola ; il fatto era divenuto proverbiale, a significare i rovesci di fortuna a cui sono soggetti anche gli uomini più potenti (cfr. ad es. Cic. Tusc. 3, 27 ; Att. 9, 9, 1).

14. Era infatti convinzione degli antichi che un'appropriata esercitazione oratoria avesse effetti benefici anche sulla salute, alla pari dell'allenamento fisico. Si veda ad es. quanto Cicerone narra di se stesso nei capp.313-316 del Brutus: da giovane egli era affetto da una summa gracilitas et infirmitas corporis, aggravata dallo sforzo fisico a cui si sottoponeva per pronunciare i suoi discorsi nel foro, al punto che alcuni medici lo avevano consigliato di abbandonare la pratica oratoria ; 
egli decise allora di mutare genus dicendi, adottando un tono di voce più disteso e moderato, e a questo scopo partì per un viaggio di istruzione in Grecia e in Asia, nel corso del quale ebbe modo di frequentare alcuni dei più celebri maestri di retorica greci, e da cui tornò in capo a due anni non modo exercitatior, sed prope mutatus, non solo con un nuovo stile oratorio, ma anche rinvigorito nel fisico (Brut. 316 nam et contentio nimia vocis resederat et quasi deferverat oratio, lateribusque vires et corpori mediocris habitus accesserat).

15. Cfr. Cic. fam. 9, 18, 2-3 quid quaeris? me quoque delectat consilium ; multa enim consequor : primum, id quod maxime nunc opus est, munio me ad haec tempora. Id cuius modi sit nescio ; tantum video, nullius adhuc consilium me huic anteponere, nisi forte melius mori fuit. [...] Sequitur illud : ipse melior fio, primum valetudine, quam intermissis exercitationibus amiseram; deinde ipsa illa, si qua fuit in me, facultas orationis, nisi me ad has exercitationes rettulissem, exaruisset. Extremum illud est, quod tu nescio an primum putes : pluris iam pavones confeci quam tu pullos columbinos, eqs. Per un'approfondita analisi di queste due lettere a Peto (fam. 9, 16 e 18), assai importanti per comprendere lo stato d'animo di Cicerone nei giorni della vittoria di Cesare, cfr. M. Demmel, Cicero und Paetus, Köln 1962, pp. 30 ss. 16. Sulla datazione e la provenienza di questa lettera cfr. le note di commento di D. R. Shackleton Bailey, Cicero, Epistulae ad familiares, ed. by D.R.S.B., II, Cambridge 1977, p. 342.

17. Il frammento citato corrisponde ad Acc. trag. 547 s. Ribb. ${ }^{3}$ L'idea espressa da Cicerone tramite questa citazione tragica anticipa quello che diverrà un luogo comune nei critici della declamazione della prima età imperiale, quando gli esercizi declamatòri vengono paragonati ai combattimenti finti affrontati dai soldati o dai gladiatori nel loro addestramento, che sono però tutt'altra cosa rispetto alle battaglie vere: si possono citare a titolo di esempio le parole dell'oratore e retore Cassio Severo riportate da Sen. contr. 3 praef. 13 deinde res ipsa diversa est : totum aliud est pugnare, aliud ventilare. Hoc ita semper habitum est, scholam quasi ludum esse, forum arenam, eqs.

18. Cfr. ThlL V.2, 508, 43 ss. ; tra gli esempi più chiari, cfr. Sen. frg. 98 Haase (dal De vita patris) si quaecumque composuit pater meus et edi voluit, iam in manus populi emisissem ; Quint. inst. praef. 1 efflagitasti ... ut libros, quos ad Marcellum meum de institutione oratoria scripseram, iam emittere inciperem ; 6 prooem. 3 eum ... librum, quem de causis corruptae eloquentiae emisi ; Plin. ep. 1, 2, 6 libelli, quos emisimus, dicuntur in manibus esse, ecc.

19. Il vitium riscontrato da Cicerone nella frase di Irzio, e castigato tramite un arguto motto di spirito, sta naturalmente nelle parole in utero, che in effetti possono apparire come un'aggiunta superflua.

20. In luogo delle parole cum is apud ipsum i manoscritti quintilianei recano lezioni prive di senso (cui sapasim cum, cusapastium, e simili) ; questa sistemazione del testo si deve a R. Volkmann, e ha buone probabilità di essere quella giusta ; L. Radermacher poneva a testo la sua congettura cum is apud Asinium, che però alla luce di quanto sappiamo delle declamazioni di Irzio e Cicerone, sembra difficilmente accettabile. Al ruolo di maestro assunto da Cicerone nei confronti di Irzio, Pansa e Dolabella, Quintiliano allude ancora in inst. 12, 11, 6 sic Pansam, Hirtium, Dolabellam more praeceptoris exercuit cotidie dicens audiensque.

21. È possibile che la battuta in questione fosse stata tratta da una raccolta di facezie ciceroniane, compilata a quanto pare dal liberto Tirone, che Quintiliano cita e utilizza nel cap. 6, 3 dell' Institutio oratoria, dedicato ai motti di spirito, e da cui attingono anche altri autori successivi (testimonianze e frammenti di questi Tulliana facete dicta sono raccolti adesso in M. Tulli Ciceronis, Fragmenta ex libris philosophicis, ex aliis libris deperditis, ex scriptis incertis, I. Garbarino recognovit, Milano 1984, pp. 128 ss., dove il nostro frammento figura con il n. 16 ; esso sarebbe comunque l'unico citato da Quintiliano al di fuori del suddetto cap. 6, 3). In ogni caso la citazione quintilianea, con la precisa menzione della circostanza a cui la battuta risale, lascia intendere che qualcosa delle declamazioni ciceroniane e dell'insegnamento retorico impartito a Irzio e agli altri discipuli si era comunque tramandato. 
22. Il frammento meriterebbe di essere incluso a sua volta nelle raccolte dei frammenti ciceroniani ; esso non compare infatti né nell'edizione delle opere complete a cura di C. F. W. Müller (M. Tulli Ciceronis scripta quae manserunt omnia, recognovit C. F. W. Müller, IV.3, Lipsiae 1879 , volume che contiene fra l'altro i fragmenta), né in quella dei soli frammenti curata più di recente da G. Garbarino (vedi la nota precedente).

23. Cfr. soprattutto J. Fairweather, Seneca the Elder cit., pp. 115 ss.; su questo problema rimando inoltre alla discussione nel mio libro Scholasticorum studia. Seneca il Vecchio e la cultura retorica e letteraria della prima età imperiale, Pisa 2007, pp. 110 ss. (con ulteriore bibliografia).

24. Causa è la parola latina con cui si traduceva il termine greco Úđó $\theta \varepsilon \sigma ı \zeta$, quel genere di

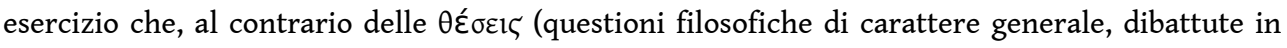
astratto e senza riferimento a persone e circostanze specifiche), prevedeva la trattazione di casi ben definiti e circostanziati (cfr. ad es. Quint. inst. 3, 5, 7 finitae autem [sc. quaestiones] sunt ex

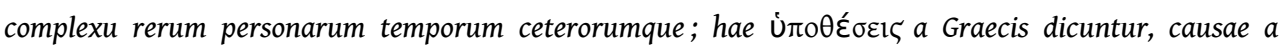

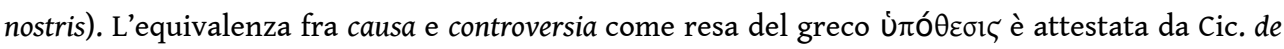
orat. 3, 109 omnem civilem orationem in horum alterutro genere versari, aut de finita controversia certis temporibus ac reis ... aut infinite de universo genere quaerentis ...; atque horum superius illud genus causam aut controversiam appellant eamque tribus, lite aut deliberatione aut laudatione, definiunt (anche se Cicerone applica tale definizione a tutti i tria genera causarum, il giudiziario, il deliberativo e l'encomiastico, e non solo alle controversiae propriamente dette); cfr. J. Fairweather, Seneca the Elder cit., pp. $125 \mathrm{~s}$.

25. Così molti dei temi declamatòri menzionati da Cicerone nei suoi trattati retorici corrispondono a quelli noti dalle raccolte di Seneca il Vecchio e dello pseudo-Quintiliano, o comunque da autori dell'età imperiale ; per fare solo un esempio, il thema di controversia citato da Cic. de orat. 2, 100 (Lex peregrinum vetat in murum ascendere; ascendit; hostis reppulit: accusatur) ricorre praticamente identico in Quintiliano (cfr. inst. 4, 4, 4 e 7, 6, 6); cfr. J. Fairweather, Seneca the Elder cit., pp. 120 s.

26. Nella lettera in questione (Cic. Att. 9, 4), scritta a poche settimane dallo scoppio della guerra civile tra Cesare e Pompeo, Cicerone confida ad Attico tutta la sua amarezza e inquietudine per l'attuale situazione politica, e gli comunica che, per non abbandonarsi completamente alla

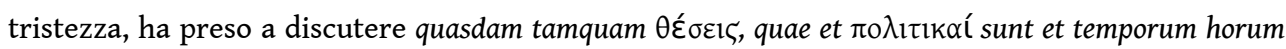

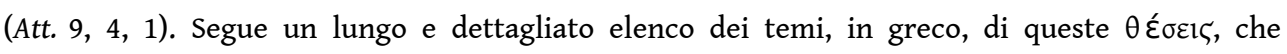
riguardano soprattutto l'atteggiamento da tenere sotto un regime tirannico (per fare solo

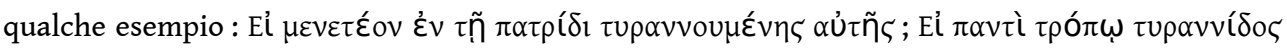

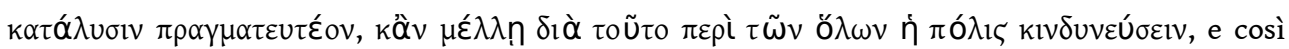
via); Cicerone conclude quindi precisando di dibattere tali questioni pro e contro, e sia in greco che in latino (Att. 9, 4, 3 in his ego me consultationibus exercens et disserens in utramque partem tum

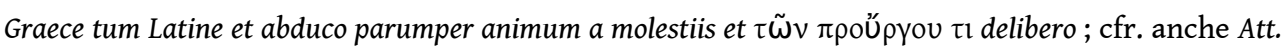
$9,9,1$ ). Sebbene anche altrove Cicerone lasci intendere di prediligere le theseis (questa è la senilis declamatio a cui allude in Tusc. 1, 7 ; cfr. anche ad Q. fr. 3, 3, 4, e J. Fairweather, op. cit., pp. 118 s.), ciò non esclude che egli abbia continuato a praticare altri tipi di esercizi retorici, come le causae 0 controversiae.

27. Così recita il thema della contr. 7, 4: Liberi parentes alant aut vinciantur. Quidam, cum haberet uxorem et ex ea filium, peregre profectus est. A piratis captus scripsit de redemptione epistulas uxori et filio. Uxor flendo oculos perdidit. Filium euntem ad redemptionem patris alumenta poscit; non remanentem alligari vult. Su questa controversia cfr. L. Beltrami, I doveri alimentari erga parentes, in R. Raffaelli, R. M. Danese, S. Lanciotti (a cura di), Pietas e allattamento filiale. La vicenda, l'exemplum, l'iconografia. Colloquio di Urbino, 2-3 maggio 1996, Urbino 1997, pp. 72-101, pp. 88 s.

28. Cfr. M. Winterbottom, The Elder Seneca, Declamations, translated by M.W., I, London 1974, p. 112, ad l. 
29. Più in generale questo modulo espressivo, che ha ben noti precedenti nella poesia epica a partire da Omero, non risulta mai attestato, a quanto ho potuto vedere, nei testi declamatòri latini ; il 'meccanismo della triplicazione' è però spesso presente nei temi delle controversiae (per limitarci alle declamazioni della raccolta senecana, tre sono i tentativi di seduzione operati dal mercator nella contr. 2, 7 ; tre le abdicationes e le successive assoluzioni ricevute dal figlio nella contr. 7, 3; tre le richieste di matrimonio rivolte dal ricco alla figlia del pauper nella contr. 8, 6 ; cfr. anche M. Lentano, L'eroe va a scuola. La figura del vir fortis nella declamazione latina, Napoli 1998, p. 110 e n. 10).

30. Da questo punto di vista si potrebbe forse avanzare l'ipotesi che la sententia fosse in greco (che Cicerone fosse solito declamare anche in greco lo abbiamo visto nelle pagine precedenti ; anche se le declamazioni dei suoi ultimi anni erano a quanto pare prevalentemente in latino). In gran parte del 1. I delle Controversiae le citazioni greche, che Seneca fa abitualmente seguire alla presentazione degli estratti dei declamatori latini, sono state volutamente tralasciate, in una fase della tradizione manoscritta precedente al nostro archetipo, da un copista che evidentemente non intendeva il greco (e anche dove esse sono state riportate, si presentano spesso in uno stato quasi irrimediabilmente corrotto); in questa contr. 1, 4 le parti in greco mancano totalmente (si veda questo stesso $\S 7$, in cui è contenuta la citazione di Cicerone, e inoltre i §§ 10-12). Si potrebbe allora supporre che le parole ter non rappresentino la traccia di un tentativo di trascrizione dei caratteri greci, subito abbandonato dal copista (questo anche in considerazione del fatto che la porzione di testo mancante sembra essere limitata alla sola sententia ciceroniana); ma si può anche pensare, e resta anzi l'ipotesi più probabile, che la lacuna sia dovuta a un normale guasto o errore meccanico.

31. Questo il thema della contr. 7, 1: Mortua quidam uxore, ex qua duos filios habebat, duxit aliam. Alterum ex adulescentibus domi parricidi damnavit, tradidit fratri puniendum: ille exarmato navigio imposuit. Delatus est adulescens ad piratas, archipirata factus est. Postea pater peregre profectus captus est ab eo et remissus in patriam. Abdicat filium. La pena prevista per i parricidi era di essere cuciti dentro un sacco di cuoio (il culleus), per poi essere gettati in mare; nella controversia il figlio rinuncia ad applicare tale pena nei confronti del fratello, e si limita a metterlo in mare su un'imbarcazione priva di tutto l'equipaggiamento (nei colores difensivi alcuni retori argomenteranno che questa era una forma di supplizio anche più spietata del culleus, in quanto costringeva il reo a vedere la sua pena, oppure che si trattava di un modo per affidare il giudizio sulla colpevolezza del frater a un'entità superiore : cfr. contr. 7, 1, 24-25). Tale situazione stimola comunque la fantasia dei declamatori, che ne traggono spunto per alcune efficaci descrizioni del navigium squassato (cfr. ad es. contr. $7,1,2 ; 8 ; 10$ ), oppure della tempesta che si immagina abbattersi sull'imbarcazione (cfr. contr. 7, 1, 4 e 26 ; su questo pezzo descrittivo topico cfr. S. F. Bonner, op. cit., p. 59).

32. Su Porcio Latrone, uno dei retori più famosi e di maggiore successo della prima età imperiale, compatriota e amico di Seneca il Vecchio (che oltre a farne il principale protagonista della sua antologia declamatoria, gli dedica un commosso ricordo nella praefatio al 1. I delle Controversiae : contr. 1 praef. 13-24), cfr. H. Bornecque, Les déclamations et les déclamateurs d'après Sénèque le Père, Lille 1902, pp. 188 ss. ; anche J. Fairweather, Seneca the Elder cit., pp. 251 ss. (in particolare sulla personalità letteraria di Latrone).

33. Cfr. soprattutto contr. 1, 1, 15-16, dove Seneca osserva che Latrone considerava un tale tipo di color, che faceva leva sul prevalere dell'adfectus sulla ratio, più potente di qualsiasi altro argomento. Lo sviluppo che Latrone dà al color nella contr. 1, 1 richiama a sua volta assai da vicino il nostro esempio (cfr. Sen. contr. 1, 1, 16 non potui, inquit, sustinere illud durum spectaculum. Offensam mihi putas tantum excidisse? Mens excidit: non animus mihi constitit, non in ministerium sustinendi corporis suffecerunt pedes, oculi subita caligine obtorpuerunt; si osservi, oltre al resto, il ricorrere in entrambi i passi del termine spectaculum). 
34. Il testo è quello di Håkanson, che pone tra cruces il tràdito ingenti, segnalando in apparato le congetture, ugualmente probabili per il senso, languenti, fugiente, oppure deficiente ; come altrove osservato dallo stesso L. Håkanson (Some Critical Notes on Seneca the Elder, « Amer. Journ. Philol. » 97, 1976, pp. 121-129, p. 127), il confronto con il § 17 (sublapsum est intercepto spiritu corpus) rende pressoché sicuro che l'ablativo spiritu debba legarsi a titubantem, e non a descripsisset, come intendevano gli editori precedenti, che mantenevano nel testo ingenti.

35. Cfr. J. Fairweather, Seneca the Elder cit., p. 169. Un color analogo ricorre anche negli estratti di altri declamatori, come Albucio Silo (cfr. contr. 7, 1, 1), e soprattutto Rubellio Blando (cfr. contr. 7, 1, 6 subito mihi non sentienti ferrum cum animo pariter excidit: torpent manus, et nescio qua perturbatione tenebrae stupentibus offunduntur oculis. Intellexi quam difficile esset parricidium facere, etiam quod imperaret pater).

36. Sulla figura e la personalità di Albucio Silo, un altro dei principali retori della prima età imperiale (alla sua presentazione è dedicata la praefatio al l. VII delle Controversiae senecane ; il suo profilo biografico si è inoltre conservato nel frammento superstite del De rhetoribus di Svetonio, cap. 30), cfr. H. Bornecque, op. cit., pp. 145 ss. ; R. A. Kaster, op. cit., pp. 313 ss.

37. Cfr. contr. 7, 1, 17 non possum fratrem occidere: pone hoc loco piratam : non poterit. Quidam occidere hominem tantum non possunt; quorundam adversus hostes deficit manus. Fratris quoque beneficium non est tam magnum, pater, quam putas: non ille te noluit occidere, sed non potuit (dove il motivo è esteso anche alla mancata uccisione del padre da parte dell'altro figlio divenuto pirata).

38. Su Gallione, giovane declamatore assai stimato da Seneca il Vecchio, a cui fu legato anche da uno speciale rapporto di amicizia (ne adottò il figlio secondogenito, Anneo Novato), cfr. H. Bornecque, op. cit., pp. 173 ss.

39. Cfr. anche R. Chambert, Pirates et voyageurs dans les Controverses de Sénèque le Père, "Rév. Étud. Lat. »77,1999, pp. 149-69, p. 160.

40. Su Musa, retore di origine greca non particolarmente apprezzato da Seneca (che gli dedica un breve ritratto in contr. 10 praef. 9-10, additandolo come vera e propria incarnazione del vitium del tumor), cfr. H. Bornecque, op. cit., p. 181.

41. Cfr. contr. 7, 1, 14-15 alii vivere sine rei publicae administratione non possunt; aliis in privato latere et extra omnem invidiam secessisse praecipua tranquillitas est; aliis non potest persuaderi ut matrimonio obligentur, aliis ut careant. Sunt qui castra timent, sunt qui cicatricibus suis gaudent. In tanta morum varietate videte quantulum sit quod excusem : non ambitioni, non inertiae veniam peto: misericors sum, non possum occidere hominem. Come si può vedere, i tre esempi di scelte di vita antitetiche proposti da Musa (concernenti l'impegno politico, il matrimonio e la vita militare) hanno ben poco a che fare con la questione dell'incapacità di uccidere, su cui il figlio basa la sua difesa ; segno evidente di come il locus communis sia qui inserito in maniera posticcia e quasi a forza.

42. Cfr. contr. 7, 1, 15 centurio Luculli Mithridaten non potuit occidere; dextra simul ac mens elanguit. Pro bone Iuppiter, Mithridaten quam non dubium parricidam !. L'exemplum in questione si rifà a un episodio poco noto della seconda guerra mitridatica, quando, dopo la sconfitta romana a Zela (67 a.c.), un centurione dell'esercito di Lucullo riuscì ad avvicinarsi a Mitridate con l'intenzione di ucciderlo, ma venuto al dunque riuscì soltanto a ferirlo a una coscia, prima di essere individuato e catturato (cfr. App. Mithr. 89, 404).

43. Oltre al già ricordato ritratto di contr. 10 praef. 9 , cfr. contr. 7, 5, 13, dove Musa è criticato proprio per l'inserimento forzato, a suggello della trattazione di un locus communis, di un exemplum completamente fuori luogo (su questi passi mi sono soffermato nel libro Scholasticorum studia cit., pp. 198 ss.).

44. Sulla figura di Cestio Pio, originario di Smirne, in Asia Minore, ma titolare a Roma di una scuola molto frequentata, anch'egli fra i retori più ammirati e più spesso citati da Seneca nella sua raccolta, cfr. H. Bornecque, op. cit., pp. 160 ss. 
45. Un'idea simile anche nel color di Rubellio Blando, citato subito prima (cfr. contr. 1, 4, 9 Blandus hoc colore: utrimque fili nomen audio: pater rem petit iustiorem, mater faciliorem. Et illud post descriptionem adiecit : fatebor vobis, parricidium coram patre facere non potui).

46. Cfr. contr. 7, 1, 20, con la discussione sull'opportunità o meno di attaccare apertamente la noverca nei colores in difesa del figlio.

47. Sull'aemulatio Ciceronis e la sua 'canonizzazione' nell'ambito delle scuole di retorica cfr. R. A. Kaster, Becoming 'CICERO', in P. Knox-C. Foss (ed.), Style and Tradition. Studies in honor of Wendell Clausen, Stuttgart-Leipzig 1998, pp. 248-63 ; sull'atteggiamento 'schizofrenico' dei declamatori nei confronti del modello ciceroniano cfr. inoltre quanto ho osservato in Scholasticorum studia cit., pp. $217 \mathrm{~s}$

\section{RIASSUNTI}

Nella quarta controversia del 1. I della raccolta Oratorum et rhetorum sententiae divisiones colores Seneca il Vecchio cita una sententia tratta da una controversia di Cicerone, l'unico frammento a noi noto di una declamazione ciceroniana. Sull'autenticità di tale frammento sono stati avanzati dei dubbi, ma l'analisi dei riferimenti all'attività declamatoria di Cicerone contenuti nella sua corrispondenza, suffragati da un passo dell'Institutio oratoria di Quintiliano, porta alla conclusione che le declamazioni ciceroniane, in particolare quelle tenute negli ultimi anni della sua vita insieme ad alcuni importanti personaggi (come Irzio e Pansa), nonostante il loro carattere privato avevano conosciuto qualche forma di circolazione presso un pubblico più vasto, tanto che la loro memoria poteva essersi conservata almeno fino all'epoca di Quintiliano. Ciò accresce la possibilità che anche il frammento trasmesso da Seneca sia autentico; e la notizia senecana assume una rilevanza più generale per la storia dello sviluppo del genere declamatorio a Roma, documentando la natura e i temi degli esercizi retorici svolti da Cicerone, che risultano essere del tutto simili a quelli praticati dai retori dell'età imperiale.

Il testo della sententia è andato perduto per un guasto della tradizione manoscritta senecana ; ma la parafrasi data da Seneca consente di ricostruirne il contenuto e di stabilire che essa sviluppava un particolare color difensivo, che forma il cardine dell'argomentazione anche in due controversiae della raccolta senecana, applicato dai singoli retori in varianti diverse, che sembrano però rifarsi tutte al precedente ciceroniano. La testimonianza di Seneca offre così un piccolo ma significativo esempio dell'aemulatio Ciceronis e della fortuna immediata di questo autore nelle scuole di retorica del primo impero.

\section{INDICE}

Mots-clés : Cicerone, colores, controversiae, declamazione, discussione dell'autenticità, epistolario di Cicerone, fortuna di Cicerone, frammenti, imitazione, scuole di retorica, Seneca il Vecchio 
AUTORE

EMANUELE BERTI

e.berti@sns.it 